\title{
Review of A psycholinguistic approach to technology and language learning
}

\author{
Tzu-Hua Chen, National Taiwan Normal University
}

Luke Plonsky, Georgetown University

A psycholinguistic approach to technology and language learning

Leow, R P., Cerezo, L., Baralt, M. (Eds.)

2015

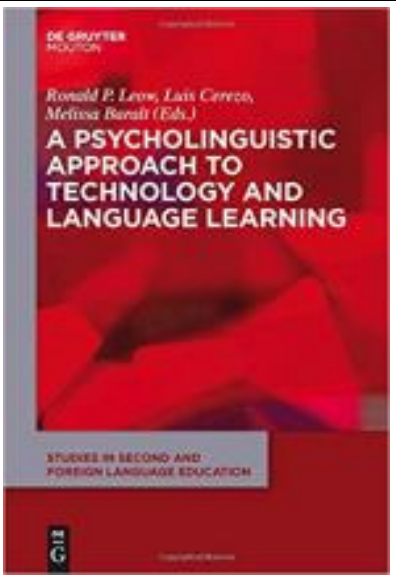

ISBN: 978-1-61451-367-4

US \$ 140.00

$267 \mathrm{pp}$.

Mouton De Gruyter

Berlin, Germany

\section{Review}

The realm of second language acquisition (SLA) has experienced a social turn that started in the mid-1990s (Block, 2003). Following this shift, SLA brought the field to "fruitful epistemological diversity that affords unique opportunities to enrich our multilayered understanding of additional language learning" (Ortega, 2011, p. 178). One such opportunity is presented in A psycholinguistic approach to technology and language learning, which is an ambitious, seminal, book-length work edited by Leow, Cerezo, and Baralt. It considers the mediation of SLA by digital technology from a psycholinguistic perspective. As stated by the editors in the preface, the major aim of this volume is to establish a dialogue between psycholinguistically-motivated theories and applications (i.e., research and pedagogy alike) to fully explore the potential of technology that mediates SLA. Thus, this book adopts a unique four-pronged approach (theoretical, methodological, empirical, and pedagogical) to technology used for SLA. In doing so, the editors hope to encourage their socially-oriented colleagues to do the same and to promote eclectic integration of the two theoretical camps into future research and practice. In addition to the major aim, the editors are especially interested in drawing attention to the potential benefit of technology to enhance second language (L2) learners' attention and cognitive processes as well as to the role that such processes play in technology-mediated SLA.

Following a brief editorial introduction, the 13 chapters of the volume are divided into four sections. Section 1, entitled Introduction to technology and SLA theory, consists of two thought-provoking chapters that lay the groundwork for this edited collection. In the introductory Chapter 1, Petersen and Sachs provide an informative overview of the opportunities and challenges of a number of popular technologies, including multimedia, synchronous computer-mediated communication (SCMC), intelligent computer-assisted language learning (ICALL), and mobile technology in networked language instruction and learning. Petersen and Sachs then advocate for active contributions by various stakeholders (i.e., through open-source sharing of teaching and research materials and technology training for practitioners) in conjunction with the advance of technology to foster communities of practice. Chapter 2 moves on to an insightful-and 
detailed - theoretical synthesis of existing CALL studies. Examining 115 empirical studies from three major CALL journals, Cerezo reports that about half of the studies (42\%) did not interpret the findings based on any existing theories, that diverse socially oriented approaches are preferred to cognitive approaches, and that $63 \%$ of CALL research draws from mainstream theories in SLA. Cerezo concludes by emphasizing the theoretical and practical contributions of a psycholinguistic perspective for CALL research, but also argues for a synergy between cognitive and socially oriented approaches.

Chapter 3 by Sanz, Morales-Front, Zalbidea, and Zárate-Sández begins the second section, Technology and SLA research: Methodological issues. This chapter reports on a survey examining how doctoral students of applied linguistics utilize computer software and equipment as well as their perceptions on the advantages and challenges of using technology for SLA research. Based on the findings, the authors provide constructive suggestions to graduate programs that include providing training on how to operate advanced equipment, promoting the use of freeware, encouraging collaboration among novice researchers from different disciplines, and organizing workshops targeting specific technologies and techniques. In Chapter 4, Leow and Suh apply Leow's (1999) internal and external validity criteria to a methodological synthesis of 17 journal studies on the comparative efficacy of CALL versus traditional offline learning in promoting vocabulary learning, reading comprehension, and listening comprehension. Focusing on violations of the validity criteria in the studies, the chapter offers some empirically-supported recommendations on overcoming limitations. Throughout the chapter, the authors highlight the importance of considering research designs and reporting practices before arriving at any conclusion on the superiority of one medium over the other (see parallel comments in Plonsky \& Ziegler, 2016). Readers who wish to improve methodological rigor in their research may find this chapter particularly helpful.

Suh's meta-analysis and research synthesis (Chapter 5) set the stage for the subsequent six empirical studies, which comprise the book's third section, Technology and SLA research: Empirical studies. Suh gives a systematic review of 11 quantitative studies published in 11 refereed journals that touch upon the comparative effectiveness of the acquisition of different types of linguistic forms (i.e., grammatical vs. lexical forms) in CALL versus in traditional offline learning environments and under different CALL treatments (i.e., SCMC vs. e-tutor vs. computerized glosses). Additionally, Suh investigates the link between study validity and L2 linguistic form learning outcomes in these chosen studies. The key finding is that CALL was better than traditional learning in promoting grammar learning. In addition to showing readers the areas which still need further research (e.g., the comparative efficacy of different types of task features and target linguistic features in CALL vs. in a traditional learning environment), the author cautions against interpretation of existing published meta-analyses in CALL while underscoring a need for robust research designs and advanced reporting practices in future empirical studies. The meta-analysis contributes to the growing body of primary and meta-analytic CALL research by continuing to address the relationship between study validity and L2 learning outcomes in CALL research (see also Plonsky, 2015; Plonsky \& Ziegler, 2016; Ziegler, 2016). This chapter flows logically from the preceding one since the importance of study validity is reinforced and further explored in relation to acquisition of L2 linguistic forms in CALL versus in traditional learning environments.

Following Suh's work, four of the six empirical studies that make up the rest of the section (Chapters 6-9) employ think-aloud protocols to examine the role of attention and cognitive processes in SLA. In Chapter 6, Medina describes a study that explores the role of both presentation mode (computer vs. paper-based) and awareness level (noticing vs. understanding) of glosses in L2 Spanish morphosyntactic development and reading comprehension. Pedagogical implications of this study are that glosses are not necessary in instructional design, as they are detrimental to text comprehension, and that morphological recognition and production can be achieved through rule discovery by learners. In Chapter 7, Hsieh revisits a previous article (Hsieh, 2008) by comparing L2 Spanish learners' level of awareness in morphosyntactic development in two types of instructional mediums: C-FTF (video-taped computerized version of face-toface instruction) and CAI (computer-assisted instruction via a virtual e-tutor in the form of a game). The unique implications of the study are that depth of processing, compared with level of awareness, is a better determinant of L2 development, and that a learner-centered instructional medium (i.e., CAI) is more 
effective than a teacher-centered instructional medium (i.e., C-FTF) for L2 morphosyntactic development.

In Chapter 8, Gurzynski-Weiss, Al Khalil, Baralt, and Leow extend a study by Sachs and Suh (2007), examining the relationship between level of awareness (high vs. low), type of implicit feedback (enhanced vs. unenhanced recasts), and type of linguistic items (lexis vs. morphology vs. syntax) in a text-based SCMC environment. In Chapter 9, de la Fuente focuses on the relative efficacy of L1 and L2 explicit metalinguistic feedback on awareness and learning of L2 Spanish forms. The study finds that L1 feedback offers L2 learners additional cognitive benefits, suggesting that CALL materials and activities should take into account the bilingual nature of L2 learners' minds. Also situating L2 development in a text-based SCMC environment, Baralt and Leow devote Chapter 10 to research the link between learner uptake, cognitive task complexity (simple vs. complex), and L2 learning of Spanish past subjunctive. Unlike findings with FTF environments, the study found that learners' uptake during both cognitively simple and complex tasks in an online environment was not related to learning. In Chapter 11, Serafini and Pennestri address the pedagogical value of learner response systems, namely clickers, as a novel way of providing feedback to L2 Spanish grammatical forms (preterit and imperfect aspectual distinction) in a classroom setting. Two variables, type of feedback (implicit vs. explicit) and timing (immediate vs. delayed), were investigated. The study demonstrated that immediate explicit feedback resulted in the best performance in grammatical forms production and that clickers are both a welcome psycholinguistic research tool and a pedagogical tool. Taken together, these studies offer invaluable insights into the connections between awareness level and SLA and generally substantiate that a high level of awareness is related to L2 development.

The last section of the volume is entitled Technology and practice. This section shifts from a focus on psycholinguistically-motivated empirical studies to a discussion of psycholinguistically-motivated CALL activities and directions for future research and practice. Chapter 12 is particularly useful for readers interested in pedagogical applications and software or game development for L2 learning. Cerezo, Moreno, and Leow start by introducing a clear-cut classification of CALL technologies in which tasks might be implemented. The authors then illustrate examples according to the 10 psycholinguistically-motivated methodological principles (MPs) of task-based language teaching (TBLT) defined by Doughty and Long (2003). The chapter concludes with some bona fide exemplars and examples of less-controlled (e.g., simulations and augmented reality games) and more-controlled CALL activities (e.g., e-tutors, new form of simulations) in detail. The final chapter, Chapter 13, pulls the volume together as the editors succinctly summarize the contents of every chapter and conclude with calls for future research and practice based on contributor insights. Specifically, the editors call for employing divergent cognitive and socially oriented approaches, particularly the former. They also advocate for the use of advanced technologies (i.e., brainbased methods and psycholinguistic tools) to understand how the L2 is attended to and for technologymediated task design as a future research program. Along with each of these calls to action, the editors reiterate the crucial role of L2 learners' attentional resources and cognitive processes in CALL.

Well written and accessible, this excellent volume accomplishes the goal that it sets out to achieve: to initiate a conversation between psycholinguistic theories and applications. The volume is well-organized, moving from more theoretical chapters to more practical ones. Perhaps one of the greatest strengths of this volume is that an array of psycholinguistically-motivated theories and concepts is introduced and applied to empirical studies as the volume unfolds, which is particularly useful for researchers new to the field. Some of the major theories, concepts, and research methods readers are exposed to in this book include the noticing hypothesis, interaction approach, TBLT, textual enhancement, form-focused instruction, cognition hypothesis, and think-aloud protocols, among others.

One potential shortcoming is the lack of research using eye-tracking methodology to study the link between L2 learners' attention and cognitive processes and SLA in CALL environments (e.g., Smith, 2012). Another methodological shortcoming relates to the research syntheses and meta-analysis in Chapters 2, 4, and 5. Specifically, if the contributors had included both published and unpublished studies, the meta-analysis and methodological and theoretical syntheses might have limited the potential for publication bias (e.g., Lin, 
2015; Ziegler, 2016). In addition, there might have been chapters that deal with learners' attention and its impact on SLA when using other technological innovations, such as mobile technology, ICALL (e.g., automatic speech recognition), and tools like concordancers, which were mentioned in Chapters 1 and 12. In doing so, the volume might have more comprehensively attained the goal of a better understanding of how L2 learners' attentional resources and cognitive processes are deployed when using digital technologies for L2 learning. However, we are aware that studies addressing the empirical link between attention and cognitive processes and SLA have just begun to bloom in the realm of CALL-SLA research, and are still scant vis-à-vis technological applications (for a parallel comment, see Chen, 2016).

Overall, A psycholinguistic approach to technology and language learning represents a much-needed contribution to the field by bringing together current and prospective theories, research methods, empirical findings, and pedagogical practice. As such, this compelling, versatile volume has great potential to drive the area forward by opening up promising avenues for further research and making pedagogical recommendations firmly grounded in research findings. Ultimately, this book is a valuable resource for experienced and novice researchers and L2 teaching practitioners, as well as for curriculum, software, and materials developers.

\section{References}

Block, D. (2003). The social turn in second language acquisition. Washington, DC: Georgetown University Press.

Chen, T.-H. (2016). Task-based L2 interaction via synchronous computer-mediated communication: A research synthesis. Poster presented at the 35th Second Language Research Forum (SLRF 2016), New York, NY, USA.

Doughty, C. J., \& Long, M. H. (2003). Optimal psycholinguistic environments for distance foreign language learning. Language Learning \& Technology, 7(3), 50-80. Retrieved from http://lit.msu.edu/vol7num3/pdf/doughty.pdf

Hsieh, H.-C. (2008). The effects of type of exposure and type of post-exposure task on L2 development. Journal of Foreign Language Instruction, 2, 117-138.

Leow, R. P. (1999). The role of attention in second/foreign language classroom research: Methodological issues. In J. Gutiérrez-Rexach \& F. Martínez-Gil (Eds.), Advances in Hispanic linguistics: Papers from the 2nd Hispanic Linguistics Symposium (pp. 60-71). Somerville, MA: Cascadilla Press.

Lin, H. (2015). A meta-synthesis of empirical research on the effectiveness of computer-mediated communication (CMC) in SLA. Language Learning \& Technology, 19(2) 85-117. Retrieved from http://lit.msu.edu/issues/june2015/lin.pdf

Ortega, L. (2011). SLA after the social turn: Where cognitivism and its alternatives stand. In D. Atkinson (Ed.), Alternative approaches to second language acquisition (pp.167-180). New York, NY: Routledge.

Plonsky, L. (2015). Quantitative considerations for improving replicability in CALL and applied linguistics. CALICO Journal, 32, 232-244.

Plonsky, L., \& Ziegler, N. (2016). The CALL-SLA interface: Insights from a second-order synthesis. Language Learning \& Technology, 20(2), 17-37. Retrieved from http://ltt.msu.edu/issues/june2016/plonskyziegler.pdf

Sachs, R., \& Suh, B.-R. (2007). Textually enhanced recasts, learner awareness, and L2 outcomes in synchronous computer-mediated interaction. In A. Mackey (Ed.), Conversational interaction in second language acquisition: A collection of empirical studies (pp. 197-227). Oxford, UK: Oxford University Press. 
Smith, B. (2012). Eye tracking as a measure of noticing: A study of explicit recasts in SCMC. Language Learning \& Technology, 16(3), 53-81. Retrieved from http://llt.msu.edu/issues/october2012/smith.pdf

Ziegler, N. (2016). Synchronous computer-mediated communication and interaction: A meta-analysis. Studies in Second Language Acquisition, 38(3), 553-586.

\section{About the Authors}

Tzu-Hua Chen recently received her MA in TESOL from National Taiwan Normal University. Her research interests include the CALL-SLA interface, SLA (e.g., interaction approach, TBLT, instructed pragmatics), research methodology, research synthesis, and meta-analysis. She has published in English Teaching \& Learning and presented or published several papers on cognitive and psycholinguistic aspects of CALL and SLA at major applied linguistics, SLA, and TESOL conferences in the US, Canada, and Taiwan.

Email: tzuhuachen2017@gmail.com

Luke Plonsky ( $\mathrm{PhD}$, Michigan State University) is an Assistant Professor of Applied Linguistics at Georgetown University where he teaches courses in SLA and research methods. His work on these and other topics has appeared in a variety of journals (e.g., Applied Linguistics, Language Learning) and edited volumes. Luke is Associate Editor of Studies in Second Language Acquisition, Managing Editor of Foreign Language Annals, and Co-Director of the IRIS Database for instruments in language learning and teaching.

Email: luke.plonsky@georgetown.edu 